\title{
Two Formal Analyses of Attack Graphs*
}

\author{
S. Jha \\ Computer Sciences Department \\ University of Wisconsin Madison, WI 53706 \\ E-mail: jha@cs.wisc.edu
}

\author{
O. Sheyner and J. Wing \\ Computer Science Department \\ Carnegie Mellon University \\ Pittsburgh, PA 15213 \\ E-mail: \{sheyner,wing $\} @$ cs.cmu.edu
}

\begin{abstract}
An attack graph is a succinct representation of all paths through a system that end in a state where an intruder has successfully achieved his goal. Today Red Teams determine the vulnerability of networked systems by drawing gigantic attack graphs by hand. Constructing attack graphs by hand is tedious, error-prone, and impractical for large systems. By viewing an attack as a violation of a safety property, we can use off-the-shelf model checking technology to produce attack graphs automatically: a successful path from the intruder's viewpoint is a counterexample produced by the model checker. In this paper we present an algorithm for generating attack graphs using model checking as a subroutine.

Security analysts use attack graphs for detection, defense and forensics. In this paper we present a minimization analysis technique that allows analysts to decide which minimal set of security measures would guarantee the safety of the system. We provide a formal characterization of this problem: we prove that it is polynomially equivalent to the minimum hitting set problem and we present a greedy algorithm with provable bounds. We also present a reliability analysis technique that allows analysts to perform a simple costbenefit trade-off depending on the likelihoods of attacks. By interpreting attack graphs as Markov Decision Processes we can use the value iteration algorithm to compute the probabilities of intruder success for each attack the graph.

Keywords: Attack graph, model checking, minimization analysis, reliability analysis, Markov Decision Processes, network vulnerability, security.
\end{abstract}

${ }^{*}$ S. Jha was supported by the Office of Naval Research under contracts N00014-01-1-0796 and N00014-01-1-0708. O. Shyener and J. Wing were supported by the Defense Advanced Research Projects Agency and the Army Research Office (ARO) under contract no. DAAD19-01-1-0485. The views and conclusions contained herein are those of the authors and should not be interpreted as necessarily representing the official policies or endorsements, either expressed or implied, of the DOD, ARO, ONR or the U.S. Government.

\section{Motivation}

As networks of hosts continue to grow, evaluating their vulnerability to attacks becomes increasingly more important to automate. When evaluating the security of a network, it is not enough to consider the presence or absence of isolated vulnerabilities. A large network builds upon multiple platforms and diverse software packages and supports several modes of connectivity. Inevitably, such a network will contain security holes that have escaped notice of even the most diligent system administrator.

To evaluate the vulnerability of a network of hosts, a security analyst must take into account the effects of interactions of local vulnerabilities and fi nd global vulnerabilities introduced by interconnections. A typical process for vulnerability analysis of a network is shown in Figure 1. First, scanning tools determine vulnerabilities of individual hosts. Using this local vulnerability information along with other information about the network, such as connectivity between hosts, the analyst produces an attack graph. Each path in an attack graph is a series of exploits, which we call atomic attacks, that leads to an undesirable state (e.g., a state where an intruder has obtained administrative access to a critical host).

\subsection{Attack Graphs and Intrusion Detection}

Attack graphs can serve as a basis for detection, defense, and forensic analysis. To motivate our study of attack graphs and attack graph generation algorithms, we discuss the potential applications of attack graphs to these areas of security.

\section{Detection}

System administrators are increasingly deploying intrusion detections systems (IDSs) to detect and combat attacks on their network. Such systems depend on software sensor modules that detect suspicious events and activity and issue alerts. Setting up the sensors usually involves a trade-off 


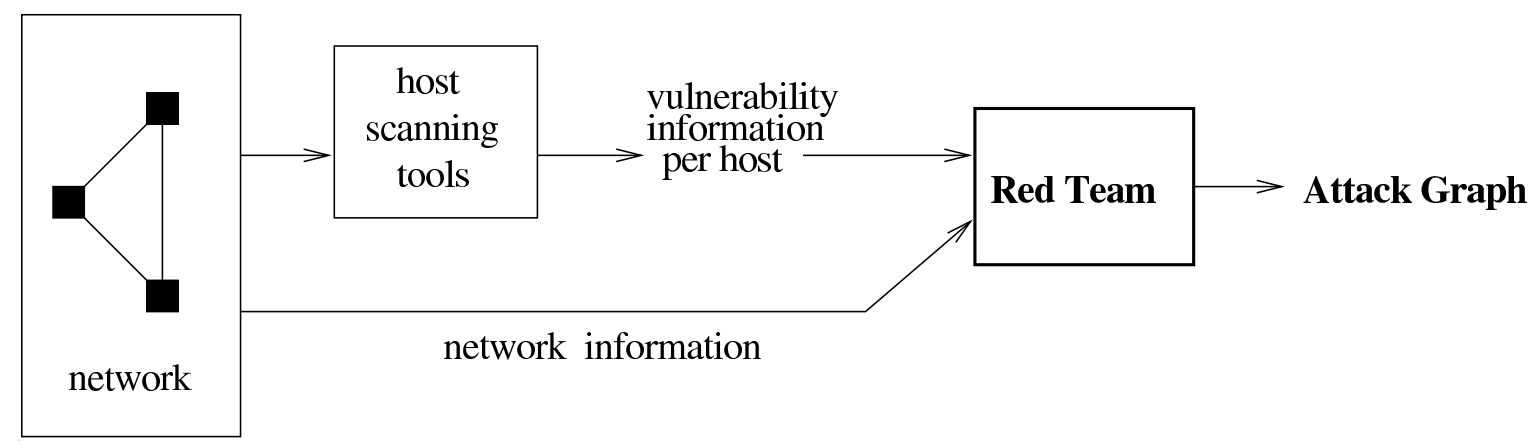

Figure 1. Vulnerability Analysis of a Network

between sensitivity to intrusions and the rate of false alarms in the alert stream. When the sensors are set to report all suspicious events, the sensors frequently issue alerts for benign background events. This often results in administrators turning off the IDS entirely. On the other hand, decreasing sensor sensitivity reduces their ability to detect real attacks.

To deal with this problem, intrusion detection systems usually employ heuristic algorithms to correlate alerts from a large pool of heterogeneous sensors. Valdes and Skinner [19] describe a probabilistic approach to alert correlation. Successful correlation of multiple alerts increases the chance that the suspicious activity indicated by the alerts is in fact malicious.

Attack graphs can enhance both heuristic and probabilistic correlation approaches. Given a graph describing all likely attacks (i.e., sequences of attacker actions), an IDS can match individual alerts to attack edges in the graph. Matching successive alerts to individual paths in the attack graphs dramatically increases the likelihood that the network is under attack. This on-line vigilance allows the IDS to predict attacker goals, aggregate alarms to reduce the volume of alert information to be analyzed, and reduce the false alarms rates. Knowledge of attacker goals and likely next steps helps guide defensive response.

\section{Defense}

A further benefi t of attack graphs is that they can help analyze potential effectiveness of intrusion detections systems offline. In Section 3 we will show how attack graphs can be generated automatically from models of the network. We will also show that we can incorporate both the security policy and the intrusion detection system in the model and generate attack graphs for specifi c network confi gurations. Attack graphs enable an administrator to perform several kinds of analysis to assess their security needs: marking the paths in the attack graph that an IDS will detect; determining where to position new IDS components for best coverage; exploring trade-offs between different security policies and between different software/hardware confi gurations; and identifying the worst-case scenarios and priori- tizing defense accordingly.

\section{Forensics}

After a break-in, forensic analysis is used to fi nd probable attacker actions and to assess damage. If legal action is desired, analysts seek evidence that a sequence of sensor alerts comprises a coherent attack plan, and is not merely a series of isolated, benign events. This task becomes even harder when the intruders obfuscate attack steps by slowing down the pace of the attack and varying specifi c steps. It has been suggested that a convincing argument as to the malicious intent of intruder actions can be constructed by matching data extracted from IDS logs to a formal reference model based on attack graphs [17].

\subsection{Our Contributions}

We have seen that constructing attack graphs is a crucial part of performing vulnerability analysis of a network of hosts. Currently, Red Teams produce attack graphs by hand, often drawing gigantic diagrams on floor-to-ceiling whiteboards. Doing this by hand is tedious, error-prone, and impractical for attack graphs larger than a hundred nodes. We have demonstrated in earlier work that model checking can be applied to automatically generate attack graphs [15]. In this paper, we show that the attack graphs produced by our method are exhaustive, i.e., covering all possible attacks, and succinct, i.e., containing only relevant states (see Section 3.2).

We also provide a formal and detailed explanation of our model. Our defi nitions are based on a fi nite-state model of the network whose state transitions are described using standard pre- and post-conditions. Each state transition corresponds to a single atomic attack by the intruder. A state in the model represents the state of the system between atomic attacks. A typical transition from state $s_{1}$ to state $s_{2}$ corresponds to an atomic attack whose preconditions are satisfi ed in $s_{1}$ and whose effects hold in state $s_{2}$. An attack is a sequence of state transitions culminating in the intruder achieving his goal. The entire attack graph is thus a repre- 
sentation of all the possible ways in which the intruder can succeed.

As already discussed, attack graphs can be used to perform a variety of analysis. Specifi cally, attack graphs can be used to answer the following questions of interest to a system administrator:

Question 1: What successful attacks are undetected by the IDS?

Question 2: If all measures in a set $M^{\prime}$ are implemented, does the network become safe (more secure)?

Question 3: Given a set of measures $M$, what is the smallest subset of measures whose implementation makes the network safe?

Answers to these questions, can help an analyst or network administrator in choosing the best upgrade strategy. These questions are addressed in Section 5.

When we are modeling a system operating in an uncertain environment, certain transitions in the model represent the system's reaction to changes in the environment. We can think of such transitions as being outside of the system's control - they occur when triggered by the environment. When no empirical information is available about the relative likelihood of such environment-driven transitions, we can model them only as nondeterministic "choices" made by the environment. Moreover, for new vulnerabilities data for estimating probabilities might not be available. However, sometimes empirical data make it possible to assign probabilities to environment-driven transitions. We would like to take advantage of such information to quantify the probabilistic behavior of attack graphs. In this context, a system administrator might be interested in the following question:

Question 4: The deployment of which security measure(s) will increase the likelihood of thwarting an attacker?

The answer to this question is provided in Section 6.1. The system administrator can use the answer to question 4 to perform a quantitative evaluation of various security fi xes (see example 1).

The main contributions of this paper over our earlier work [15] are:

- This paper explores the semantics of our network model more formally.

- In our earlier work [15] we proved that fi nding a minimum set of atomic attacks which must be removed to thwart an intruder is NP-complete. In this paper, we further explore the complexity of this problem. Section 5.1 proves that the problem is polynomially equivalent to the minimum hitting set problem where the collection of sets is represented as a labeled directed graph. This reduction provides us additional insight, which enabled us to fi nd a greedy algorithm with prov- able bounds, which can be used to answer questions 1,2 , and 3 .

- This paper also presents an algorithm to compute the reliability (which is defi ned as the likelihood of an intruder not succeeding) for a network. A desirable feature of our algorithm is that it allows for incomplete information, i.e., probabilities of all transitions need not be provided. To our knowledge, previous metrics in the area of security require complete information.

Related work is provided in Section 2. Section 3 describes our model and our model checking based algorithm to generate attack graphs. An example network is presented in Section 4. Section 4 also describes our network model in detail. This network is used throughout the paper for illustrative purposes. In Section 5 we present analysis which helps us answers questions 1,2, and 3. Section 6 describes probabilistic attack graphs and our algorithm to compute reliability. Answer to question 4 is provided in that section. The proof of correctness of our algorithm to compute reliability are based on Markov Decision Processes and presented in the companion technical report [9]. Finally, directions for future work and concluding remarks are presented in Section 7.

\section{Related Work}

Phillips and Swiler [12] propose a concept of attack graphs that is very similar to the one described here. However, they take an "attack-centric" view of the system. Since we work with a general input language, in our model seemingly benign system events (such as failure of a link and user errors) and attacks occur simultaneously. Therefore, our attack graphs are more general than the one proposed by Phillips and Swiler. Based on these ideas a tool for generating attack graphs is presented in [18]. The tool constructs the attack graph by forward exploration starting from the initial state. A symbolic model checker (like NuSMV) works backward from the goal state to construct the attack graph. A major advantage of the backward algorithm is that vulnerabilities that are not relevant to the safety property (or the goal of the intruder) are never explored. This can result in signifi cant savings in space. In fact, Swiler et al. [18] refer to the advantages of the backward search in their paper. Moreover, by using model checking we leverage off all the sophisticated reduction techniques developed in that area. The post-facto analysis suggested by Phillips and Swiler is also different from the one presented in this paper. We plan to incorporate their analysis into our tool.

Dacier [6] proposes the concept of privilege graphs, where each node represents a set of privileges owned by the user and arcs represent vulnerabilities. Privilege graphs are 
then explored to construct attack state graphs, which represents different ways in which an intruder can reach a certain goal, such as root access on a host. Based on the attack state graphs a metric, called the mean effort to failure or METF, is proposed. An experimental evaluation of their framework is described by Orlato et al. [11]. At the surface our notion of attack graphs seem similar to the one proposed by Dacier. However, as is the case with Phillips and Swiler, Dacier again takes an "attack-centric" view of the world. As pointed out earlier, our attack graphs are more general. From the experiments conducted by Orlato et al. it appears that even for small examples the space required to construct attack state graphs becomes prohibitive. Model checking has made signifi cant advances in representing large state spaces. Therefore, by basing our algorithm on model checking we leverage off those advances and can hope to represent large attack graphs. However, the analytical analysis proposed by Dacier can also be performed on attack graphs constructed by our tool. We also plan to conduct an experimental evaluation similar to the one performed by Orlato et al.

Ritchey and Amman [14] also used model checking for vulnerability analysis of networks. They used the unmodifi ed model checker SMV [16]. Therefore, they could only obtain one counter-example or one attack corresponding to a intruder's goal. In contrast, we modifi ed the model checker NuSMV to produce complete attack graphs, which represents all possible attacks. We also described analysis that can be performed on these attack graphs. These analysis techniques cannot be meaningfully performed on single attacks.

\section{Generating Attack Graphs using Model Checking}

First, we formally defi ne attack graphs, the data structure used to represent all possible attacks on our networked system. We restrict our attention to attack graphs representing violations of safety properties ${ }^{1}$.

Definition 1 Let $A P$ be a set of atomic propositions. An attack graph or $A G$ is a tuple $G=\left(S, \tau, S_{0}, S_{s}, L\right)$, where $S$ is a set of states, $\tau \subseteq S \times S$ is a transition relation, $S_{0} \subseteq S$ is a set of initial states, $S_{s} \subseteq S$ is a set of success states, and $L: S \rightarrow 2^{A P}$ is a labeling of states with a set of propositions true in that state. Intuitively, $S_{s}$ denotes intruder's goals, e.g., obtaining root access on a critical host.

Unless stated otherwise, we assume that the transition relation $\tau$ is total. We defi ne an execution fragment as a fi nite sequence of states $s_{0} s_{1} \ldots s_{n}$ such that $\left(s_{i}, s_{i+1}\right) \in \tau$ for all $0 \leq i<n$. An execution fragment with $s_{0} \in S_{0}$ is

\footnotetext{
${ }^{1}$ We say more on liveness properties in Section 7
}

an execution, and an execution whose fi nal state is in $S_{s}$ is an attack, i.e., the execution corresponds to a sequence of atomic attacks leading to the intruder's goal state.

Next we turn our attention to algorithms for automatic generation of attack graphs. Starting with a description of a network model $M$ and a security property $p$, the task is to construct an attack graph representing all executions of $M$ that violate $p$-these are the successful attacks. For the kinds of attack graph analysis suggested in Section 1, it is essential that the graphs produced by the algorithms be exhaustive and succinct. An attack graph is exhaustive with respect to a model $M$ and correctness property $p$ if it covers all possible attacks in $M$ leading to a violation of $p$, and succinct if it only contains those states of $M$ from which the system can get to a state violating $p$.

\subsection{Reachability Analysis}

If we restrict ourselves to safety properties, an attack graph may be constructed by performing a simple statespace search. Starting with the initial states of the model $M$, we use a graph traversal procedure (e.g., depth-fi rst search) to fi nd all reachable success states where the safety property $p$ is violated. The attack graph is the union of all paths from initial states to success states.

While this algorithm has the advantage of simplicity, it handles only safety properties and may run into the state explosion problem for non-trivial models. Model checking has dealt with both of these issues with some success, so we will consider algorithms based on that technology.

\subsection{Model Checking Algorithm}

Model checking is a technique for checking whether a formal model $M$ of a system satisfi es a given property $p$. In our work, we use the model checker NuSMV [10], for which the model $M$ is a fi nite labeled transition system and $p$ is a property expressed in Computation Tree Logic $(C T L)$. For now, we consider only safety properties, which in CTL have the form AG $f$ (i.e., $p=\mathbf{A G} f$, where $f$ is a formula in propositional logic). If the model $M$ satisfi es the property $p$, NuSMV reports "true." If $M$ does not satisfy $p$, NuSMV produces a counter-example. A single counterexample shows an execution that leads to a violation of the property. In this section, we explain how to construct attack graphs for safety properties using model checking.

Attack graphs depict ways in which the system can reach an unsafe state (or, equivalently, a successful state for the intruder). We can express the property that an unsafe state cannot be reached as:

$$
\mathbf{A G}(\neg \text { unsafe })
$$

When this property is false, there are unsafe states that are reachable from the initial state. The precise meaning of 


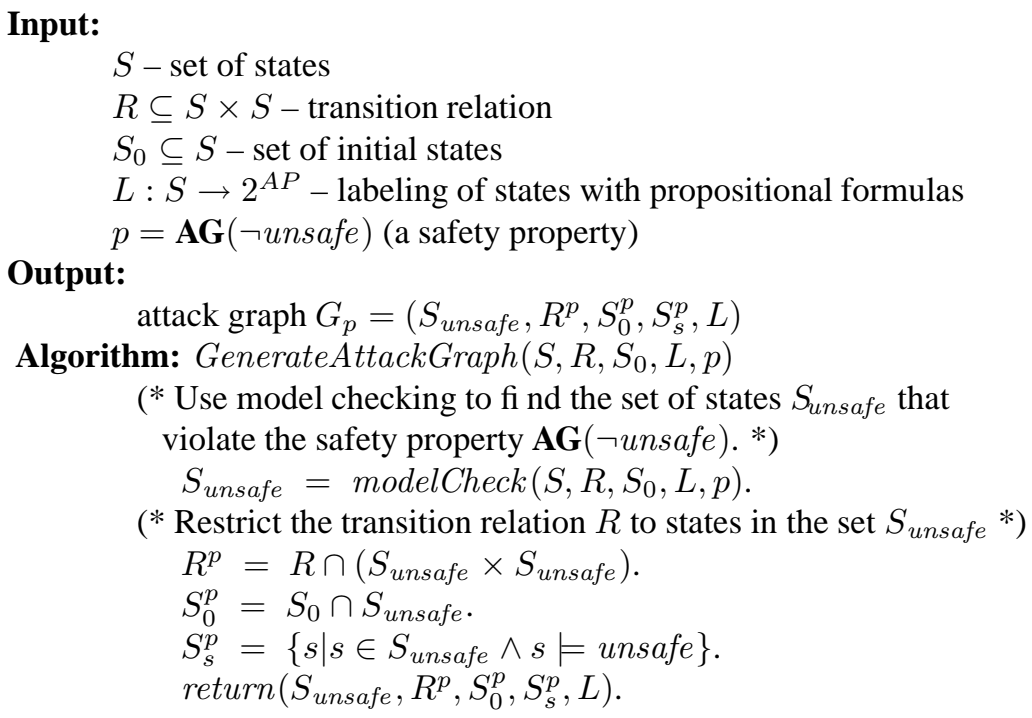

\section{Figure 2. Algorithm for Generating Attack Graphs}

unsafe depends on the application. For example, in the network security example given in Section 4, the property given below is used to express that the privilege level of the intruder on the host with index 2 should always be less than the root (administrative) privilege.

\section{AG(network.adversary.privilege[2] < network.priv.root)}

We briefly describe the algorithm for constructing attack graphs for the property AG( $\neg$ unsafe). The fi rst step is to determine the set of states $S_{r}$ that are reachable from the initial state. Next, the algorithm computes the set of reachable states $S_{\text {unsafe }}$ that have a path to an unsafe state. The set of states $S_{\text {unsafe }}$ is computed using an iterative algorithm derived from a fi x-point characterization of the AG operator [4]. Let $R$ be the transition relation of the model, i.e., $\left(s, s^{\prime}\right) \in R$ if and only if there is a transition from state $s$ to $s^{\prime}$. By restricting the domain and range of $R$ to $S_{\text {unsafe }}$ we obtain a transition relation $R^{p}$ that represents the edges of the attack graph. Therefore, the attack graph is $\left(S_{\text {unsafe }}, R^{p}, S_{0}^{p}, S_{s}^{p}, L\right)$, where $S_{\text {unsafe }}$ and $R^{p}$ represent the set of nodes and edges of the graph, respectively, $S_{0}^{p}=S_{0} \cap S_{\text {unsafe }}$ is the set of initial states, and $S_{s}^{p}=\left\{s \mid s \in S_{\text {unsafe }} \wedge s \models\right.$ unsafe $\}$ is the set of success states. This algorithm is given in Figure 2.

In symbolic model checkers, such as NuSMV, the transition relation and sets of states are represented using BDDs [3], a compact representation for boolean functions. There are effi cient BDD algorithms for all operations used in the algorithm shown in Figure 2.

\subsection{Attack Graph Properties}

We can show that an attack graph $G$ generated by the algorithm in Figure 2 is exhaustive (Lemma 1(a)) and succinct with respect to states and edges (Lemma 1(b) and 1(c)). Proof of the following lemma is straightforward and follows from the defi nitions.

Lemma 1 The following properties of the attack graph $G$ are true:

(a) exhaustive

An execution $e$ of the input model $\left(S, R, S_{0}, L\right)$ violates the property $p=\mathbf{A G}(\neg$ unsafe $)$ if and only if $e$ is an attack in the attack graph $G=\left(S_{\text {unsafe }}, R^{p}, S_{0}^{p}, S_{s}^{p}, L\right)$.

(b) succinct with respect to states

A state $s$ of the input model $\left(S, R, S_{0}, L\right)$ is in the attack graph $G$ if and only if there is an attack in $G$ that contains $s$.

(c) succinct with respect to edges

An edge $t=\left(s_{1}, s_{2}\right)$ of the input model $\left(S, R, S_{0}, L\right)$ is in the attack graph $G$ if and only if there is an attack in $G$ that includes $t$.

\section{A Simple Intrusion Detection Example}

Consider the example network shown in Figure 3. There are two target hosts, $i p_{1}$ and $i p_{2}$, and a fi rewall separating them from the rest of the Internet. As shown, each host is running two of three possible services (ftp, sshd, a database). An intrusion detection system (IDS) monitors the network traffi $\mathrm{c}$ between the target hosts and the outside 


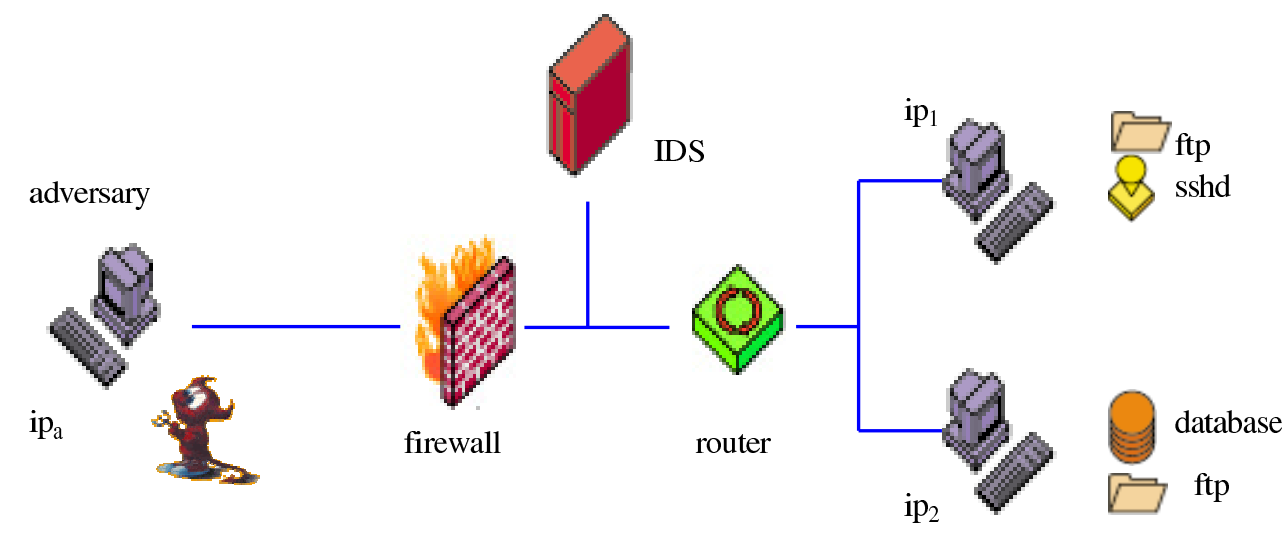

Figure 3. Example Network

world. There are four possible atomic attacks, identifi ed numerically as follows: (0) sshd buffer overflow, (1) ftp .rhosts, (2) remote login, and (3) local buffer overflow. If an atomic attack is detectable, the intrusion detection system will trigger an alarm; if an attack is stealthy, the IDS misses it. The ftp .rhosts attack needs to fi nd the target host with two vulnerabilities: a writable home directory and an executable command shell assigned to the ftp user name. The local buffer overflow exploits a vulnerable version of the xterm executable.

In this section, we construct a fi nite state model of the example network so that each state transition corresponds to a single atomic attack by the intruder. A state in the model represents the state of the system between atomic attacks. A typical transition from state $s_{1}$ to state $s_{2}$ corresponds to an atomic attack whose preconditions are satisfi ed in $s_{1}$ and whose effects hold in state $s_{2}$.

The intruder launches his attack starting from a single computer, $i p_{a}$, which lies outside the fi rewall. His eventual goal is to disrupt the functioning of the database. For which, the intruder needs root access on the database host $i p_{2}$.

\subsection{States of the Finite State Machine Model}

\section{The Network}

We model the network as a set of facts, each represented as a relational predicate. The state of the network specifi es services, host vulnerabilities, connectivity, and a remote login trust relationship between hosts. There are six boolean variables for each host, specifying whether any of the three modeled services are running and whether any vulnerabilities are present on that host.

\begin{tabular}{|l||l|}
\hline variable & meaning \\
\hline \hline $\operatorname{ssh}_{h}$ & $\begin{array}{l}\text { ssh service is running } \\
\text { on host } h\end{array}$ \\
\hline $\mathrm{ftp}_{h}$ & $\begin{array}{l}\text { ftp service is running } \\
\text { on host } h\end{array}$ \\
\hline data $_{h}$ & $\begin{array}{l}\text { database is running } \\
\text { on host } h\end{array}$ \\
\hline $\operatorname{wdir}_{h}$ & $\begin{array}{l}\text { ftp home directory is } \\
\text { writable on host } h\end{array}$ \\
\hline fshell $_{h}$ & $\begin{array}{l}\text { ftp user has executable } \\
\text { shell on host } h\end{array}$ \\
\hline xterm $_{h}$ & $\begin{array}{l}\text { xterm executable is } \\
\text { vulnerable to overflow on host } h\end{array}$ \\
\hline
\end{tabular}

Connectivity is expressed as a ternary relation $R \subseteq$ Host $\times$ Host $\times$ Port, where $R\left(h_{1}, h_{2}, p\right)$ means that host $h_{2}$ is reachable from host $h_{1}$ on port $p$. The constants $s p$ and $f p$ will refer to the specifi c ports for the ssh and ftp services, respectively. Slightly abusing notation, we write $R\left(h_{1}, h_{2}\right)$ when there is a network route from $h_{1}$ to $h_{2}$. Similarly, we model trust as a binary relation RshTrust $\subseteq$ Host $\times$ Host, where $\operatorname{RshTrust}\left(h_{1}, h_{2}\right)$ indicates that a user may log in from host $h_{2}$ to host $h_{1}$ without authentication (i.e., host $h_{1}$ "trusts" host $h_{2}$ ).

\section{The Intruder}

The function $p l v l_{A}:$ Hosts $\rightarrow\{$ none, user, root $\}$ gives the level of privilege that intruder $A$ has on each host. There is a total order on the privilege levels: none $<$ user $<$ root.

Several state variables specify which attack the intruder will attempt next: 


\begin{tabular}{|l||l|}
\hline variable & meaning \\
\hline \hline attack & attack type \\
\hline source & source host \\
\hline target & target host \\
\hline strain & stealthy/detectable attack \\
\hline
\end{tabular}

Intrusion Detection System

Atomic attacks are classifi ed as being either detectable or stealthy with respect to the Intrusion Detection System (IDS). If an attack is detectable, it will trigger an alarm when executed on a host or network segment monitored by the IDS; if an attack is stealthy, the IDS does not detect it.

We specify the IDS with a function ids: Host $\times$ Host $\times$ Attack $\rightarrow\{d, s, b\}$, where $i d s\left(h_{1}, h_{2}, a\right)=d$ if attack $a$ is detectable when executed with source host $h_{1}$ and target host $h_{2} ; i d s\left(h_{1}, h_{2}, a\right)=s$ if attack $a$ is stealthy when executed with source host $h_{1}$ and target host $h_{2}$; and $i d s\left(h_{1}, h_{2}, a\right)=b$ if attack $a$ has $b$ oth detectable and stealthy strains, and success in detecting the attack depends on which strain is used. When $h_{1}$ and $h_{2}$ refer to the same host, $i d s\left(h_{1}, h_{2}, a\right)$ specifi es the intrusion detection system component (if any) located on that host. When $h_{1}$ and $h_{2}$ refer to different hosts, $i d s\left(h_{1}, h_{2}, a\right)$ specifi es the intrusion detection system component (if any) monitoring the network path between $h_{1}$ and $h_{2}$. In addition, a global boolean variable specifi es whether the IDS alarm has been triggered by any previously executed atomic attack.

\subsection{Initial States}

Initially, there is no trust between any of the hosts; the trust relation $\operatorname{Tr}$ is empty. The connectivity relation $R$ is shown in the following table. An entry in the table corresponds to a pair of hosts $\left(h_{1}, h_{2}\right)$. Each entry is a triple of boolean values. The first value is ' $y$ ' if $h_{1}$ and $h_{2}$ are connected by a physical link, the second value is ' $y$ ' if $h_{1}$ can connect to $h_{2}$ on the ftp port, and the third value is ' $\mathrm{y}$ ' if $h_{1}$ can connect to $h_{2}$ on the sshd port.

\begin{tabular}{|l||c|c|c|}
\hline$R$ & $i p_{a}$ & $i p_{1}$ & $i p_{2}$ \\
\hline \hline$i p_{a}$ & $\mathrm{y}, \mathrm{n}, \mathrm{n}$ & $\mathrm{y}, \mathrm{y}, \mathrm{y}$ & $\mathrm{y}, \mathrm{y}, \mathrm{n}$ \\
\hline$i p_{1}$ & $\mathrm{y}, \mathrm{n}, \mathrm{n}$ & $\mathrm{y}, \mathrm{y}, \mathrm{y}$ & $\mathrm{y}, \mathrm{y}, \mathrm{n}$ \\
\hline$i p_{2}$ & $\mathrm{y}, \mathrm{n}, \mathrm{n}$ & $\mathrm{y}, \mathrm{y}, \mathrm{y}$ & $\mathrm{y}, \mathrm{y}, \mathrm{n}$ \\
\hline
\end{tabular}

We use the connectivity relation to reflect the fi rewall rule sets as well as the existence of physical links. For the table above, the fi rewall is open and does not place any restrictions on the flow of network traffi c.

Initially, the intruder has root privileges on his own machine $i p_{a}$ and no privileges on the other hosts.

The paths between $\left(i p_{a}, i p_{1}\right)$ and between $\left(i p_{a}, i p_{2}\right)$ are monitored by a single network-based IDS. The path between $\left(i p_{1}, i p_{2}\right)$ is not monitored. There are no other hostbased intrusion detection components. The IDS detects the remote login attack, and the detectable strains of the sshd buffer overflow attack.

\subsection{Transitions}

Our model has nondeterministic state transitions. If the current state of the network satisfi es the preconditions of more than one atomic attack rule, the intruder nondeterministically "chooses" one of those attacks. The state then changes according to the effects clause of the chosen attack rule. The intruder repeats this process until his goal is achieved.

We model four atomic attacks. Throughout the description, $S$ is used to designate the source host and $T$ the target host. $R(S, T, p)$ denotes that host $T$ is reachable from host $S$ on port $p$.

\section{Sshd Buffer Overflow}

This remote-to-root attack immediately gives a remote user a root shell on the target machine.

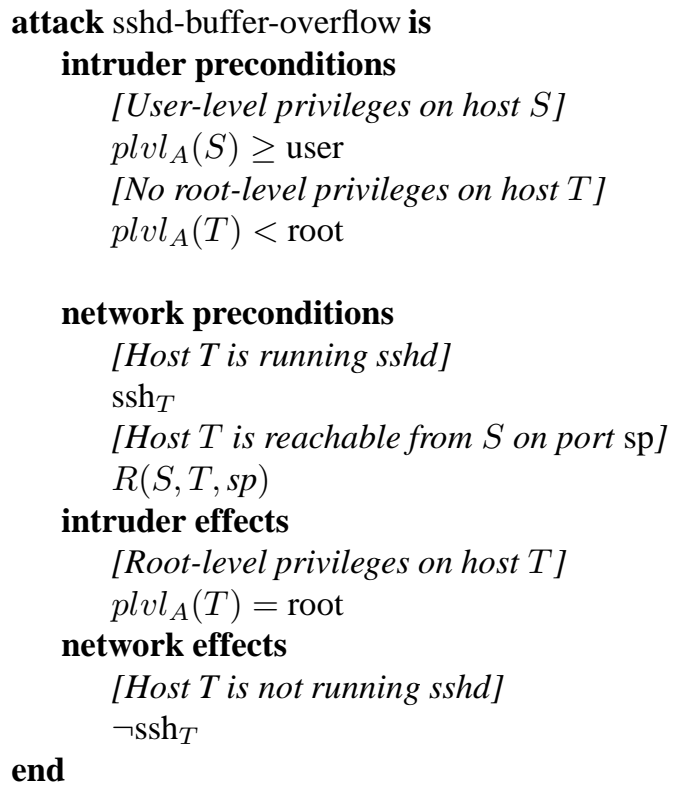

\section{Ftp .rhosts}

Using an ftp vulnerability, the intruder creates an .rhosts fi le in the ftp home directory, creating a remote login trust relationship between his machine and the target machine.

attack ftp-rhosts is intruder preconditions

[User-level privileges on host $S$ ]

$\operatorname{plvl}_{A}(S) \geq$ user 


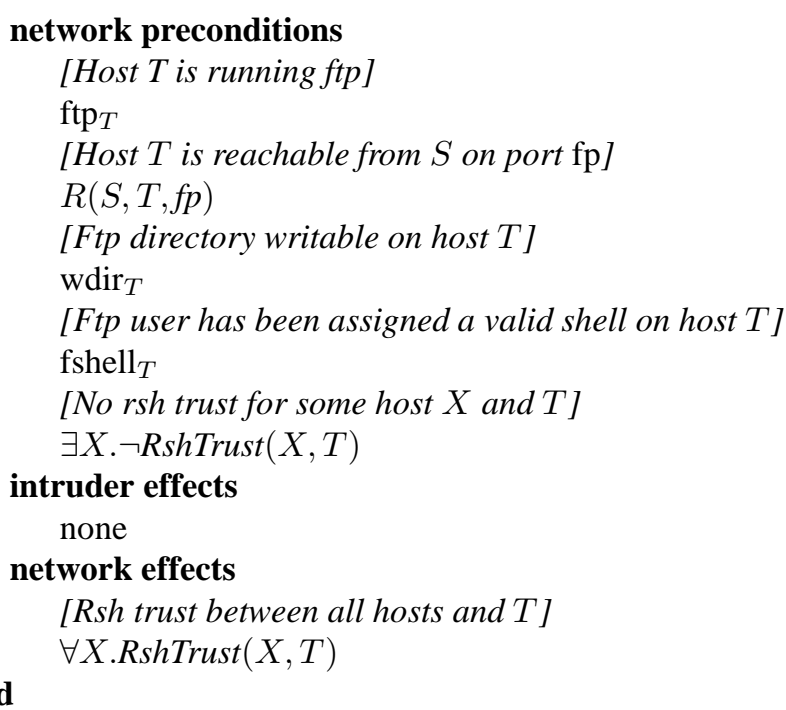

\section{Remote Login}

Using an existing remote login trust relationship between two machines, the intruder logs in from one machine to another, getting a user shell without supplying a password. This operation is usually a legitimate action performed by regular users, but from the intruder's viewpoint, it is an atomic attack.

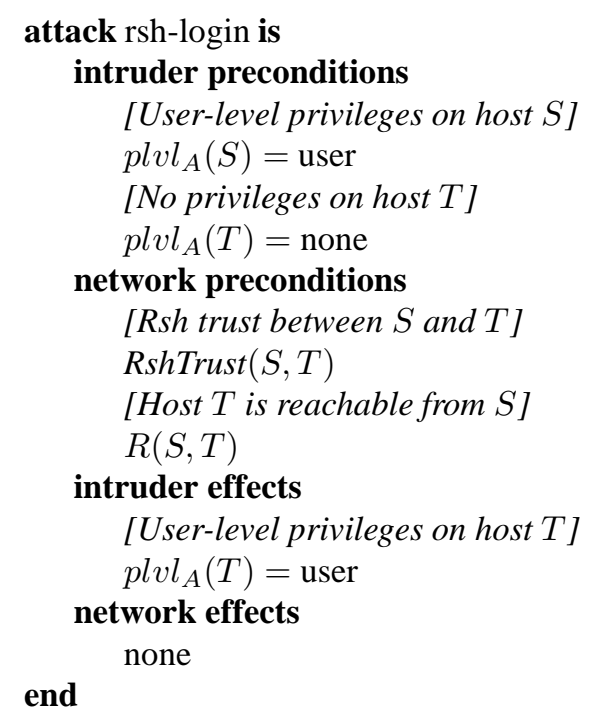

\section{Local Buffer Overflow}

If the intruder has acquired a user shell on the target machine, the next step is to exploit a buffer overflow vulnerability on a setuid root file to gain root access. attack local-setuid-buffer-overflow is

intruder preconditions

[User-level privileges on host $T$ ]

$\operatorname{plvl}_{A}(T)=$ user

network preconditions

[There is a vulnerable xterm executable] $\mathrm{xterm}_{T}$

intruder effects

[Root-level privileges on host $T$ ]

$\operatorname{plvl}_{A}(T)=$ root

network effects

none

end

It is easy to see that each atomic attack strictly increases either the intruder's privilege level on the target host or remote login trust between hosts. This means that the attack graph has no cycles.

From our fi nite model we can now automatically construct attack graphs that demonstrate how the intruder can violate various security properties. Suppose we want to generate all attacks that demonstrate how the intruder can gain root privilege on host $i p_{2}$ and remain undetected by the IDS. The CTL formula that expresses the safety property that the intruder on host with id 2 always has privilege level below root or is detected is expressed using the following CTL property:

\section{$\mathbf{A G}$ (network.adversary.privilege[2] < network.priv.root network.detected)}

Figure 4 shows the attack graph produced by our tool for this property. Each node is labeled by an attack id number, which corresponds to the atomic attack to be attempted next; a flag S/D indicates whether the attack is stealthy or detectable by the intrusion detection system; and the numbers of the source and target hosts $\left(i p_{a}\right.$ corresponds to host number 0).

Any path in the graph from the root node to a leaf node shows a sequence of atomic attacks that the intruder can employ to achieve his goal while remaining undetected. For instance, the path highlighted by double-boxed nodes consists of the following sequence of four atomic attacks: overflow sshd buffer on host 1, overwrite .rhosts fi le on host 2 to establish rsh trust between hosts 1 and 2, log in using rsh from host 1 to host 2 , and fi nally, overflow a local buffer on host 2 to obtain root privileges.

We have also expanded the example described above by adding two additional hosts, four additional atomic attacks, several new vulnerabilities, and flexible fi rewall confi gurations. For the larger example the attack graph has 5948 nodes and 68364 edges. 


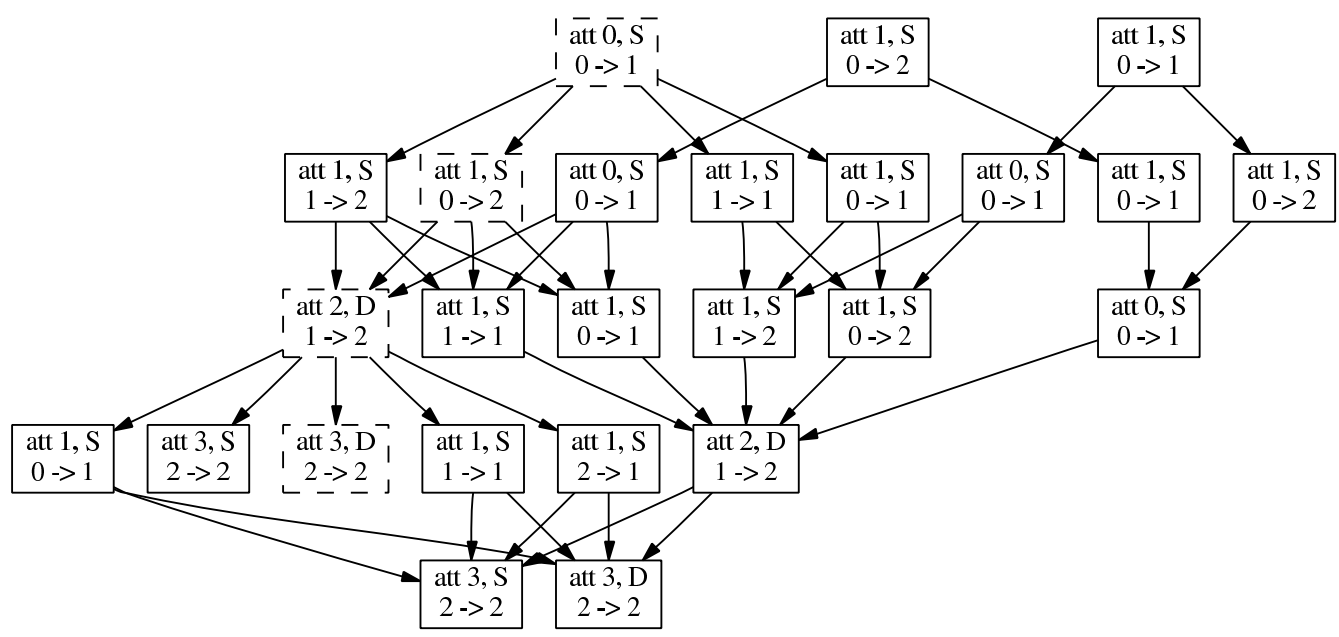

Figure 4. Attack Graph

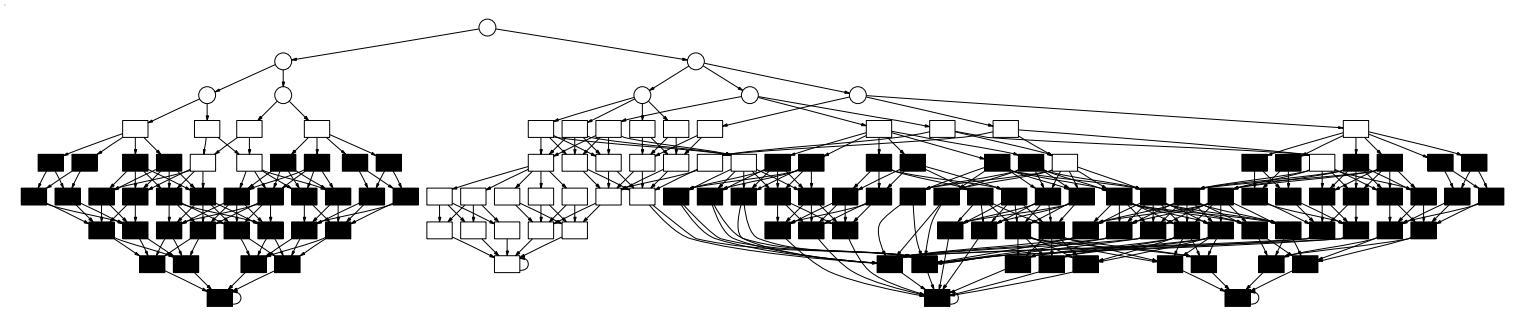

Figure 5. Attack Graph Analysis

\section{Minimization Analysis}

Once we have an attack graph generated for a specific network with respect to a given safety property, we can utilize it for further analysis. For example, an analyst may be faced with a choice between deploying additional network attack detection tools or prevention techniques. An analyst has a set of measures, such as deploying additional network detection tools or upgrading the software, available to him. This section provides answers to questions 1,2, and 3 posed in Section 1.2.

Question 1: What successful attacks are undetected by the IDS?

Answer:

To answer this question, we modify the model slightly, making only a subset of atomic attacks available to the intruder. For simplicity, we nondeterministically decide which subset to consider initially, before any attack begins; once the choice is made, the subset of available atomic attacks is fi xed during any given attack. We ran the model checker on the modifi ed model with the invariant property that says the intruder never obtains root privilege on host $i p_{2}$ :

AG(network.adversary.privilege[2] < network.priv.root)

The post-processor marked the states where the intruder has been detected by the IDS. The result is shown in Figure 5. The white rectangles indicate states where the attacker had not yet been detected by the intrusion detection system. The black rectangles are states where the intrusion detection system has sounded an alarm. Thus, white leaf nodes are desirable for the attacker because his objective is achieved without detection. Black leaf nodes are less desirable - the attacker achieves his objective, but the alarm goes off.

The resolution of which atomic attacks are available to the intruder happens in the circular nodes near the root of the graph. The fi rst transition out of the root (initial) state picks the subset of attacks that the intruder will use. Each child of the root node is itself the root of a disjoint subgraph where the subset of atomic attacks chosen for that child is used. Note that the number of such subgraphs descending from the root node corresponds to the number of subsets of atomic attacks with which the intruder can be successful- 
the model checker determines that for any other possible subset, there is no possible successful sequence of atomic attacks.

The root of the graph in Figure 5 has two subgraphs, corresponding to the two subsets of atomic attacks that will allow the intruder to succeed. In the left subgraph the sshd buffer overflow attack is not available to the intruder; it can be readily seen that the intruder can still succeed, but cannot do so while remaining undetected by the IDS. In the right subgraph, all attacks are available. Thus, the entire attack graph implies that all atomic attacks other than the sshd attack are indispensable: the intruder cannot succeed without them. The analyst can use this information to guide decisions on which network defenses can be profi tably upgraded.

The white cluster in the middle of the fi gure is isomorphic to the attack graph presented in Figure 4; it shows attacks in which the intruder can achieve his objective without detection (i.e., all paths by which the intruder reaches a white leaf in the graph).

We proceed to provide answers to questions 2 and 3 . However, fi rst we defi ne an attack graph whose edges are labeled with atomic attacks, which is produced by our postprocessor. Such an attack graph is used to perform further analysis to answer questions 2 and 3 . Assume that we have produced an attack graph corresponding to the following safety property:

$$
\mathbf{A G}(\neg \text { unsafe })
$$

Let $\mathcal{A}$ be the set of atomic attacks, and $G=$ $\left(S, E, s_{0}, s_{s}, L\right)$ be the attack graph, where $S$ is the set of states, $E \subseteq S \times S$ is the set of edges, $s_{0} \in S$ is the initial state, $s_{s} \in S$ is the success state for the intruder, and $L: E \rightarrow \mathcal{A} \cup\{\epsilon\}$ is a labeling function where $L(e)=a$ if an edge $e=\left(s \rightarrow s^{\prime}\right)$ corresponds to an atomic attack $a$, otherwise $L(e)=\epsilon$. Edges labeled with $\epsilon$ represent system transitions that do not correspond to an atomic attack. Moreover, as demonstrated below additional $\epsilon$ edges can be also introduced by our construction. Without loss of generality we can assume that there is a single initial and success state. For example, consider an attack graph with multiple initial states $s_{0}^{1}, \cdots, s_{0}^{j}$ and success states $s_{s}^{1}, \cdots, s_{s}^{u}$. We can add a new initial state $s_{0}$ and a new success state $s_{s}$ with $\epsilon$-labeled edges $\left(s_{0}, s_{0}^{m}\right)(1 \leq m \leq j)$ and $\left(s_{s}, s_{s}^{t}\right)$ $(1 \leq t \leq u)$. Suppose we are given a fi nite set of measures $M=\left\{m_{1}, \cdots, m_{k}\right\}$ and a function covers $: M \rightarrow 2^{\mathcal{A}}$. An atomic attack $a \in \operatorname{covers}\left(m_{i}\right)$ if adopting measure $m_{i}$ removes the atomic attack $a$.

Question 2: If all measures in a set $M^{\prime}$ are implemented, does the network become safe (more secure)?

\section{Answer:}

A network administrator wants to fi nd out whether adopting measures from a set $M^{\prime} \subseteq M$ will make the network safe. This question can be answered in linear time us- ing the attack graph $G$. First, we defi ne covers $\left(M^{\prime}\right)$ as $\bigcup_{m \in M^{\prime}}$ covers $(m)$. Next, we remove all edges $e$ from $G$ such that $L(e) \in \operatorname{covers}\left(M^{\prime}\right)$. The network is safe iff the success state $s_{s}$ is not reachable from the initial state $s_{0}$. This simple reachability question can be answer in time that is linear in the size of the graph.

Question 3: Given a set of measures $M$, what is the smallest subset of measures whose implementation makes the network safe?

\section{Answer:}

A network administrator wishes to fi nd a subset $M^{\prime} \subseteq M$ of smallest size, such that adopting the measures in the set $M^{\prime}$ will make the network safe. Unfortunately, this problem is $N P$-complete, but we develop good approximation algorithms. We proceed in two steps:

\section{- Step 1 (Finding a small set of atomic attacks.)}

In this step, we fi nd a set of atomic attacks whose removal makes the network safe. Checking every possible subset of attacks is exponential in the number of attacks. In a related paper [15], we show that fi nding the minimum set of atomic attacks which must be removed to thwart an intruder is in fact $N P$-complete. We also demonstrated how a minimal set can be found in polynomial-time. In this paper, we further explore the complexity of this problem. Section 5.1 proves that the problem of fi nding a minimum set of attacks is polynomially equivalent to the minimum hitting set problem, where the collection of sets is represented as a labeled directed graph. This reduction provides us additional insight, which enabled us to fi nd a greedy algorithm with provable bounds.

\section{- Step 2 (Finding a small set of measures)}

Assume that we fi nd a set of atomic attacks $A^{\prime}$ whose removal makes the network safe, or equivalently thwarts the intruder. Recall that $M=\left\{m_{1}, \cdots, m_{k}\right\}$ is the set of measures and covers $: M \rightarrow 2^{\mathcal{A}}$ is a function, where covers $\left(m_{i}\right)$ represents the set of atomic attacks that are removed by adopting the measure $m_{i}$. With each attack $a$ in the set $A^{\prime}$, we associate a set of measures $M(a)$ which is $\left\{m_{i} \mid a \in \operatorname{covers}\left(m_{i}\right)\right\}$. The set of attacks $A^{\prime}$ defi nes a collection $C_{A^{\prime}}$ of subsets of $M$. We wish to fi nd the smallest subset $M^{\prime} \subseteq M$ such that for all $a \in A^{\prime}$ there exists an $m_{i} \in M^{\prime}$ such that $a \in \operatorname{covers}\left(m_{i}\right)$, or equivalently $M^{\prime} \cap M(a) \neq \emptyset$. This is known as the minimum hitting set problem, which is NP-complete, but good approximation algorithms exist to solve this problem (see Section 5.2) 


\subsection{The Minimum Critical Attack Sets and the Minimum Hitting Set Problem}

This section addresses the fi rst step in the answer to question 3. Assume that we are given an attack graph $G=$ $\left(S, E, s_{0}, s_{s}, L\right)$, where $S$ is the set of states, $E \subseteq S \times S$ is the set of edges, $s_{0} \in S$ is the initial state, $s_{s} \in S$ is the success state for the intruder, and $L: E \rightarrow \mathcal{A} \cup\{\epsilon\}$ is a labeling function.

Given a state $s \in S$, a set of attacks $C$ is critical with respect to $s$ if and only if the intruder cannot reach his goal from $s$ when the attacks in $C$ are removed from his arsenal. Equivalently, $C$ is critical with respect to $s$ if and only if every path from $s$ to the success state $s_{s}$ has at least one edge labeled with an attack $a \in C$.

A critical set corresponding to a state $s$ is minimum (denoted by $M(s)$ ) if there is no critical set $M^{\prime}(s)$ such that $\left|M^{\prime}(s)\right|<|M(s)|$. In general, there can be multiple minimum sets corresponding to a state $s$. Of course, all minimum critical sets must be of the same size.

A critical set of an attack graph $G=\left(S, E, s_{0}, s_{s}, L\right)$ is defined as a critical set corresponding to the initial state $s_{0}$. Therefore, the Minimum Critical Set of Attacks (MCSA) problem is the problem of fi nding a minimum critical set of attacks $M\left(s_{0}\right)$. The decision version of the problem is defi ned as follows: given an attack graph $G=$ $\left(S, E, s_{0}, s_{s}, L\right)$ and a positive integer $K$, is there a critical set of attacks $A \subseteq \mathcal{A}$ such that $|A| \leq K$.

Assume that we are give an attack graph $G=$ $\left(S, E, s_{0}, s_{s}, L\right)$. A path $\pi$ is sequence of states $q_{1}, \cdots, q_{n}$, such that $q_{i} \in S$ and $\left(q_{i}, q_{i+1}\right) \in E$. A complete path starts from the initial state $s_{0}$ and ends in the success state $s_{s}$. The label of a path $\pi=q_{1}, \cdots, q_{n}$ (abusing notation, we will denote it also as $L(\pi)$ ) is a subset of a set of attacks $\mathcal{A}$

$$
\bigcup_{i=1}^{n-1}\left\{L\left(q_{i}, q_{i+1}\right)\right\} \backslash\{\epsilon\} .
$$

$L(\pi)$ represents the set of atomic attacks used on the path $\pi$. A set of attacks $A \subseteq \mathcal{A}$ is called realizable in the attack graph $G$ iff there exists a complete path $\pi$ in $G$ such that $L(\pi)=A$. In other words, an intruder can use the set of attacks $A$ to start from the initial state and reach the success state. The set of all realizable sets in an attack graph $G$ is denoted by $\operatorname{Rel}(G)$. The following lemma is easy to prove and follows straight from the defi nitions.

Lemma 2 Assume that we are give an attack graph $G=$ $\left(S, E, s_{0}, s_{s}, L\right)$. A set of attacks $A$ is critical iff

$$
\forall A^{\prime} \in \operatorname{Rel}(G) \cdot A^{\prime} \cap A \neq \emptyset .
$$

In other words, all realizable sets have a non-empty intersection with a critical set $A$.

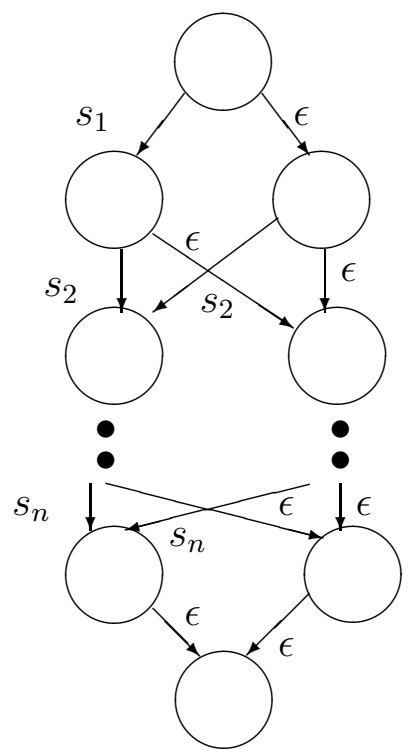

\section{Figure 6. Attack graph representing an expo- nential number of realizable sets.}

Definition 2 Hitting Set [8, Problem SP8]

Instance: Collection $C$ of subsets of a fi nite set $S$, positive integer $K \leq|S|$.

Question: Is there a subset $S^{\prime} \subseteq S$ with $\left|S^{\prime}\right| \leq K$ such that $S^{\prime}$ contains at least one element from each subset in $C$ ?

The discussion given in appendix A proves that the problem of fi nding critical sets in an attack graph is polynomially equivalent to fi nding hitting sets for a collection, with one caveat-the collection of sets $C$ is represented as an attack graph. An attack graph can be an exponentially succinct representation of a collection of sets. Figure 6 shows an attack graph of linear size whose set of realizable sets is the power set of $\left\{s_{1}, \cdots, s_{n}\right\}$. Therefore, the minimum critical set problem is polynomially equivalent to the hitting set problem where the collection of sets $C$ is represented as a labeled directed graph.

\subsection{A Greedy Algorithm}

Next, we describe a greedy algorithm GREEDYHITTING-SET for the minimum hitting set problem. Let $(C, S, K)$ be an instance of the hitting set problem. Let $S^{\prime}$ and $C^{\prime}$ be initially the empty set. The greedy algorithm executes the following steps until $C^{\prime}=C$.

- Pick an element $s$ out of the set $S \backslash S^{\prime}$ that covers the maximum number of sets in the collection $C \backslash C^{\prime}$. An element $s$ is said to cover a set $S_{1} \subseteq S$ iff $s \in S_{1}$. 
- Let $s$ be the element picked in the previous step and $C(s)$ be the collection of sets in $C$ covered by $s$. Update $S^{\prime}$ and $C^{\prime}$ as follows:

$$
\begin{aligned}
& S^{\prime} \leftarrow S^{\prime} \cup\{s\} \\
& C^{\prime} \leftarrow C^{\prime} \cup C(s)
\end{aligned}
$$

Let $H_{d}$ be the $d$-th harmonic number $\sum_{i=1}^{d} \frac{1}{i}$, and $C(s)$ be the number of sets in the collection $C$ that are covered by the element $s$.

Lemma 3 GREEDY-HITTING-SET is a polynomialtime $\rho(n)$-approximation algorithm, where $\rho(n)=$ $H\left(\max _{s \in S}\{|C(s)|\}\right)$.

The proof of the lemma follows from the equivalence between the minimum hitting set and the minimum cover problem [2] and the proof of the approximation factor $\rho(n)$ for the greedy algorithm for the minimum cover problem [5]. Using the equivalence between the problems of fi nding a minimum critical set and a minimum hitting, set we can construct a greedy procedure (called GREEDYCRITICAL-SET) for finding a critical set for the attack graph. Assume that we are given an attack graph $G=$ $\left(S, E, s_{0}, s_{s}, L\right)$, where $S$ is the set of states, $E \subseteq S \times S$ is the set of edges, $s_{0} \in S$ is the initial state, $s_{s} \in S$ is the success state for the intruder, and $L: E \rightarrow \mathcal{A} \cup\{\epsilon\}$ is a labeling function. Moreover, assume that we can compute in polynomial time the function $\mu_{G}: \mathcal{A} \rightarrow \aleph$, where $\mu_{G}(a)$ is the number of realizable sets in the attack graph $G$ that contain the attack $a$. Formally, $\mu_{G}(a)$ is equal to

$$
\mid\left\{A^{\prime} \mid a \in A^{\prime} \text { and } A^{\prime} \in \operatorname{Rel}(G)\right\} \mid .
$$

Initially, let $A^{\prime}$ be the empty set and $G^{\prime}=G$. The greedy algorithm GREEDY-CRITICAL-SET executes the following steps until $G^{\prime}$ is empty.

- Pick and element $a$ from the set $\mathcal{A} \backslash A^{\prime}$ that maximizes $\mu_{G^{\prime}}(a)$.

- Let $a$ be the element picked in the previous step. Update $A^{\prime}$ and $G^{\prime}$ as follows:

$$
\begin{aligned}
& A^{\prime} \leftarrow A^{\prime} \cup\{a\} \\
& \text { Remove all edges labeled with } a \text { from } G^{\prime}
\end{aligned}
$$

Lemma 4 GREEDY-CRITICAL-SET is a polynomialtime $\rho(n)$-approximation algorithm, where $\rho(n)=$ $H\left(\max _{a \in \mathcal{A}}\left\{\mu_{G}(a)\right\}\right)$.

Next, we explore conditions when the function $\mu_{G}$ can be computed in polynomial time. Assume that the attack graph $G$ is a DAG. An argument for this was given in Section 4.3. Moreover, assume that each atomic attack is used only once on a path from the initial state $s_{0}$ to the success state $s_{s}$. This is not a unreasonable assumption because the attack graph edges are labeled with instantiations of attack templates shown in Section 4.3, e.g., a local-setuid-bufferoverflow attack on two different hosts are distinct in the attack graph. Such attack graphs are called use once DAGs. The following lemma is easy to prove.

Lemma 5 For an attack graph that is a use once DAG, the function $\mu_{G}$ can be computed in time that is linear in size of the attack graph.

\section{Probabilistic Analysis of Attack Graphs}

One way to incorporate probabilities into attack graphs is to choose a subset of states and make transitions out of those states probabilistic. Suppose that the graph has a state $s$ with only two outgoing transitions. In a regular attack graph, the choice of which transition to take when the system is in state $s$ is nondeterministic. However, we may have some empirical data that enables us to estimate that whenever the system is in state $s$, on average it will take one of the transitions four times out of ten and the other transition six remaining times. We can place probabilities 0.4 and 0.6 on the corresponding edges in the attack graph. Intuitively, probability of the transition $s \rightarrow s^{\prime}$ represents the likelihood that the atomic attack corresponding to the transition will succeed. We call a state with known probabilities for outgoing transitions probabilistic. When we have assigned all known probabilities in this way, we are left with an attack graph that has some probabilistic and some nondeterministic states in it. We call such mixed attack graphs probabilistic attack graphs. We use probabilistic attack graphs to evaluate the reliability of a network. Note that probabilities of all the transitions might not be available because of lack of data, e.g., a new type of atomic attack.

Since the attack graph includes only those states and transitions that can lead to success states, it excludes some transitions that exist in the complete model $M$. These excluded transitions can have non-zero probability, so that the sum of probabilities of transitions from a probabilistic state will be less than 1 . To address this problem, we must model the rest of $M$ in some way. We add a "catch-all" escape state $s_{e}$ to the attack graph. A probabilistic state $s$ in the attack graph will have a transition to $s_{e}$ if and only if in $M$ there is a transition from $s$ to some state not in the attack graph. The probability of going from $s$ to $s_{e}$ will be 1 minus the sum of the probabilities of going to other states. There are no transitions out of $s_{e}$ except a self-loop (which preserves the totality of the transition relation $\tau$ ).

In an attack graph containing the escape state $s_{e}$ attacks are allowed to terminate in $s_{e}$. We will call them escape attacks, or attacks that were pre-empted by the intruder before he reached his goal. 
Definition 3 A probabilistic attack graph or PAG is a tuple $G=\left(S_{n}, S_{q}, s_{e}, S, \tau, \pi, S_{0}, S_{s}, L\right)$, where $S_{n}$ is a set of nondeterministic states, $S_{q}$ is a set of probabilistic states, $s_{e} \in S_{n}$ is a nondeterministic escape state $\left(s_{e} \notin S_{s}\right), S=$ $S_{n} \cup S_{q}$ is the set of all states, $\tau \subseteq S \times S$ is a transition relation, $\pi: S_{q} \rightarrow S \rightarrow \Re$ are transition probabilities, $S_{0} \subseteq S$ is a set of initial states, $S_{s} \subseteq S$ is a set of success states, and $L: S \rightarrow 2^{A P}$ is a labeling of states with a set of propositions true in that state.

A probabilistic attack graph (PAG) distinguishes between nondeterministic states (set $S_{n}$ ) and probabilistic states (set $S_{q}$ ). Moreover, the sets of nondeterministic and probabilistic states are disjoint $\left(S_{n} \cap S_{q}=\emptyset\right)$. The function $\pi$ specifi es probabilities of transitions from probabilistic states, so that for all transitions $s_{1} \rightarrow s_{2} \in \tau$ such that $s_{1} \in S_{q}$, we have $P\left(s_{1} \rightarrow s_{2}\right)=\pi\left(s_{1}\right)\left(s_{2}\right)>0$. Thus, $\pi(s)$ can be viewed as a probability distribution on next states. Intuitively, when the system is in a nondeterministic state $s_{n}$, we have no information about the relative probabilities of the possible next transitions. When the system is in a probabilistic state $s_{q}$, it will choose the next state according to probability distribution $\pi\left(s_{q}\right)$.

Complete probability case. Assume that each transition in the attack graph is assigned a probability, i.e., there are no nondeterministic states. Let $G=\left(S, \tau, S_{0}, S_{s}, L\right)$ be the attack graph and $P$ a function that assigns probabilities to transitions. The probabilities can be loosely interpreted as the probability of the atomic attack corresponding to the transition succeeding. We are interested in fi nding the reliability of the attack graph, i.e., the probability that the intruder will not succeed. We can view $G$ as a Markov chain with $S$ as its state space and $P\left(s_{1} \rightarrow s_{2}\right)$ as its transition probability. Let $U: S \rightarrow \Re_{+}$be the steady state probability of the Markov chain (see [7] for defi nitions and technical conditions). In this case, the reliability of the attack graph $G$ is given by the following expression:

$$
1-\sum_{s \in S_{s}} U(s)
$$

In other words, the reliability is the probability that in the "long run" the Markov chain will not be in a state in the set $S_{s}$. We wish to perform similar analysis on probabilistic attack graphs in the presence nondeterministic states.

\subsection{Reliability}

Assume that we are given a PAG $G=$ $\left(S_{n}, S_{q}, s_{e}, S, \tau, \pi, S_{0}, S_{s}, L\right)$. Intuitively, we are interested in finding out the probability that the intruder will reach a success state starting from one of the initial states. Recall that in the absence of nondeterministic states we can compute this metric by using the steady state probabilities of the Markov chain. In presence of nondeterministic states the intruder will choose transitions in order to maximize his probability of succeeding. For example, if an intruder reaches a nondeterministic state $s$ with transitions to $s_{1}, \cdots, s_{k}$, he will choose to transition to state $s_{i}(1 \leq i \leq n)$ which will maximize his probability of reaching a success state. This idea can be "formalized" using concepts from the theory of Markov Decision Processes [1,13].

Given a state $s$, the set of successors of $s$ is denoted by $\operatorname{succ}(s)$. Formally, $\operatorname{succ}(s)$ is equal to $\left\{s^{\prime} \mid\left(s, s^{\prime}\right) \in \tau\right\}$. First, we defi ne a value function $V: S \rightarrow \Re^{+}$. For all $s \in S_{s}, V(s)=1.0$. For all states $s \in S \backslash S_{s}$ the value function is iterated according to the following equations until convergence.

$V(s)= \begin{cases}\max _{s^{\prime} \in \operatorname{succ}(s)} V\left(s^{\prime}\right) & \text { if } s \in S_{n} \backslash S_{s} \\ \sum_{s^{\prime} \in \operatorname{succ}(s)} P\left(s \rightarrow s^{\prime}\right) V\left(s^{\prime}\right) & \text { if } s \in S_{q} \backslash S_{s}\end{cases}$

Let $V^{\star}$ be the value function after convergence. Intuitively, $\sum_{s \in S_{0}} V^{\star}(s)$ is the probability for the intruder to reach a success state if he "resolves" the nondeterminism to maximize the probability of succeeding. Therefore, the worst case reliability of the network is $1-\sum_{s \in S_{0}} V^{\star}(s)$. This algorithm is known as value iteration. The justifi cation of the value iteration algorithm is presented in the companion technical report [9].

Example 1 We implemented the value iteration algorithm in our attack graph post-processor and ran it on a slightly modifi ed version of the intrusion detection example from Section 4. In the modifi ed example, each attack has both detectable and stealthy variants. The intruder chooses which atomic attack to try next, and he has a certain probability of picking a stealthy or a detectable variant. We assigned imaginary probabilities of picking a stealthy attack variant as follows: 0.2 for sshd buffer overflow, 0.5 for $\mathrm{ftp}$.rhosts, 0.05 for the remote login, and 0.8 for local buffer overflow. The intruder's goal is to obtain root access on host $i p_{2}$ while remaining undetected. Accordingly, the states where this goal has been achieved were assigned benefi t value 1.0.

In this setup, the computed probability of intruder success is 0.2 , and his best strategy is to attempt sshd buffer overflow on host $i p_{1}$, and then conduct the rest of the attack from that host. The only possibility of detection is the sshd buffer overflow attack itself, since the IDS does not see the activity between hosts $i p_{1}$ and $i p_{2}$.

Given this context, a system administrator can ask the following question:

Question 4: The deployment of which security measure(s) will increase the likelihood of thwarting an attacker?

\section{Answer:}

Installing an additional IDS component to monitor the network traffi c between hosts $i p_{1}$ and $i p_{2}$ reduces the probability of the intruder remaining undetected to 0.025 ; installing 
a host-based IDS on host $i p_{2}$ reduces the probability to 0.16 . Other things being equal, this is an indication that the former remedy is more effective.

\section{Summary of Contributions and Future Work}

Our foremost contribution is the automatic generation of attack graphs. Our key insight is that an attack is equivalent to a counterexample produced by off-the-shelf model checkers; the attack/counterexample is a witness to a violation of a safety property. By a small, but critical enhancement to an existing model checker, we can easily produce attack graphs automatically; moreover, these graphs are succinct and exhaustive. A by-product of this part of our work is showing, by example, what level of abstraction is appropriate for modeling attacks. We use simple state machine specifi cations to model not just intruder behavior (by a set of atomic attacks), but also normal system behavior, system administrator recovery actions, and connectivity (communication) between subsystems.

Our second most important contribution is support for a range of formal analyses of attack graphs. Security analysts use attack graphs informally for attack detection, defense, and forensics. In this paper, we explain how they can now use our minimization analysis technique on attack graphs to more precisely answer questions like "Which security measure should I deploy in order to thwart this set of attacks?" and "Which set of security measures should I deploy to guarantee the safety of my system?" We also explain (briefly in text and in detail in the technical report [9]) how to use probabilities to perform reliability analysis. By annotating attack graphs with probabilities, we can interpret them as Markov Decision Processes (MDP). Then, by using MDP algorithms such as value iteration, security analysts can more precisely answer questions like "Which attack will incur the most damage to my system?" and "Will deploying this intrusion detection system increase or decrease the likelihood of thwarting this type of attack?"

On the theoretical front, we plan to exploit the full power of model checking by exploring how to handle liveness properties, not just safety properties. For example, a property that states a user can always access a particular file server would be violated if the server is disabled due to a denial-of-service attack. On the practical front, we plan to conduct larger case studies to illustrate the usefulness of automatically generating attack graphs. We also intend to build a tool that merges our work on attack graphs with existing intrusion detection technologies. The tool is intended help security analysts evaluate and enhance the security of a network.

\section{References}

[1] E. Altman. Constrained Markov Decision Processes. Chapman \& Hall/CRC, 1999.

[2] G. Ausiello, A. D'Atri, and M. Protasi. Structure preserving reductions among convex optimization problems. Journal of Computational System Sciences (JCSS), 21:136-153, 1980.

[3] R. E. Bryant. Graph-based algorithms for boolean function manipulation. IEEE Trans. Comput., C-35(8):677-691, Aug. 1986.

[4] E. M. Clarke, O. Grumberg, and D. Peled. Model Checking. MIT Press, 2000.

[5] T. Cormen, C. Leiserson, and R. Rivest. Introduction to Algorithms. The MIT Press, 1991.

[6] M. Dacier. Towards Quantitative Evaluation of Computer Security. PhD thesis, Institut National Polytechnique de Toulouse, December 1994.

[7] R. Durrett. Probability: Theory and Examples. Duxbury Press, 2nd edition, 1995.

[8] M. Garey and D. Johnson. Computers and Intractibility. W.H. Freeman and Company, 1979.

[9] S. Jha, O. Sheyener, and J. M. Wing. Two formal analyses of attack graphs. Technical Report CMU-CS-02-109, Carnegie Mellon University, February 2002.

[10] NuSMV: a new symbolic model checker. http://afrodite.itc.it:1024/ nusmv/.

[11] R. Ortalo, Y. Deswarte, and M. Kaaniche. Experimenting with quantitative evaluation tools for monitoring operational security. IEEE Transactions on Software Engineering, 25/5:633-650, Sept/Oct 1999.

[12] C. Phillips and L. Swiler. A graph-based system for network vulnerability analysis. In ACM New Security Paradigms Workshop, pages 71-79, 1998.

[13] M. Puterman. Markov Decision Processes-Discrete Stochastic Dynamic Programming. John Wiley \& Sons, Inc., New York, 1994.

[14] R. Ritchey and P. Ammann. Using model checking to analyze network vulnerabilities. In Proceedings of IEEE Symposium on Security and Privacy, pages 156 -165, May 2001.

[15] O. Sheyner, J. Haines, S. Jha, R. Lippmann, and J. Wing. Automated generation and analysis of attack graphs. In Proceedings of IEEE Symposium on Security and Privacy, May 2002.

[16] SMV: a symbolic model checker. http://www2.cs.cmu.edu/modelcheck/.

[17] P. Stephenson. Using formal methods for forensic analysis of intrusion events - a preliminary examination. White Paper, available at http://www.imfgroup.com/Document Library.html.

[18] L. P. Swiler, C. Phillips, D. Ellis, and S. Chakerian. Computer-attack graph generation tool. In Proceedings of the DARPA Information Survivability Conference and Exposition, June 12-14 2000.

[19] A. Valdes and K. Skinner. Probabilistic alert detection. In Recent Advances in Intrusion Detection (RAID), 2001. 


\section{A Appendix to Section 5}

Assume that we are given an attack graph $G=$ $\left(S, E, s_{0}, s_{s}, L\right)$. Moreover, suppose one can compute the set of realizable sets $\operatorname{Rel}(G)$. Lemma 2 proves that the problem of fi nding whether the attack graph $G$ has a critical set of size $\leq K$ is the hitting set problem with $C=\operatorname{Rel}(G)$, $S=\mathcal{A}$, and $K$.

Next suppose we have an instance $(C, S, K)$ of the hitting set problem. We will construct and attack graph $G^{\prime}=$ $\left(S^{\prime}, E^{\prime}, s_{0}^{\prime}, s_{s}^{\prime}, L^{\prime}\right)$, where $L^{\prime}: E^{\prime} \rightarrow S \cup\{\epsilon\}$, i.e., the set of attacks used in the attack graph $G^{\prime}$ is $S$. Moreover, the set of realizable sets $\operatorname{Rel}\left(G^{\prime}\right)$ of the graph $G^{\prime}$ is the collection $C$. A critical set of size $\leq K$ of the attack graph $G^{\prime}$ is a hitting set for the collection $C$. Next, we describe the construction of $G^{\prime}$. Let $C=\left\{C_{1}, \cdots, C_{m}\right\}$ be the collection of sets and $S=\left\{s_{1}, \cdots, s_{n}\right\}$ be the set. We make $m$ copies $S^{1}, \cdots, S^{m}$ of the set $S$. The set of elements in $S^{i}$ will be denoted by $\left\{s_{1}^{i}, \cdots, s_{n}^{i}\right\}$. The set of states $S^{\prime}$ in the attack graph $G^{\prime}$ is

$$
\left\{s_{0}^{\prime}, s_{s}^{\prime}\right\} \cup S^{1} \cup \cdots \cup S^{m} .
$$

The initial state is $s_{0}^{\prime}$ and the fin nal state is $s_{s}^{\prime}$. The set of edges $E^{\prime}$ and the labeling function $L^{\prime}$ are defi ned as follows:

- There is an edge from $s_{0}^{\prime}$ to every state in the set $\left\{s_{1}^{1}, s_{1}^{2}, \cdots, s_{1}^{m}\right\}$, and label of the edge $\left(s_{0}^{\prime}, s_{i}^{1}\right)$ is $s_{1}$ if $s_{1} \in C_{i}$, otherwise it is $\epsilon$.

- For all $1 \leq i \leq m$ and $1 \leq j \leq n-1$, there is an edge $\left(s_{j}^{i}, s_{j+1}^{i}\right)$, and the label of edge $\left(s_{j}^{i}, s_{j+1}^{i}\right)$ is $s_{j+1}$ if $s_{j+1} \in C_{i}$, otherwise it is $\epsilon$.

- There is an edge from every state in the set $\left\{s_{n}^{1}, s_{n}^{2}, \cdots, s_{n}^{m}\right\}$ to the state $s_{s}^{\prime}$, and labels of all these edges is $\epsilon$.

The sizes of the sets $S^{\prime}$ and $E^{\prime}$ in the attack graph $G^{\prime}$ are $m n+2$ and $2 m+m n$ respectively. It is easy to see that $\operatorname{Rel}\left(G^{\prime}\right)$ is equal to $C$, and $S^{\prime} \subseteq S$ is a critical set of the attack graph $G^{\prime}$ iff $S^{\prime}$ is a hitting set for the collection $C$. Since the size of $G^{\prime}$ is polynomial in the size of the instance of the hitting set problem and the hitting set problem is $N P$-complete, the MCSA problem is $N P$-hard. Lemma 1 in [15] proves that MCSA is in NP. Therefore, MCSA is $N P$-complete. The next example illustrates our construction.

Note: The discussion given above also proves that the problem of fi nding a minimum set of measures whose adoption will make the network safe is also $N P$-complete. One can simply take the set of measures $M$ to be the set of attacks $\mathcal{A}$.

Example 2 We give a short example to illustrate the reduction. Consider a set $S=\left\{s_{1}, s_{2}, s_{3}\right\}$. Suppose that the collection $C$ consists of the following subsets:

$$
\begin{aligned}
& C_{1}=\left\{s_{1}, s_{2}\right\} \\
& C_{2}=\left\{s_{2}, s_{3}\right\} \\
& C_{3}=\left\{s_{2}\right\}
\end{aligned}
$$

The attack graph $G^{\prime}$ corresponding to this problem is shown in Figure 7. The set of attacks is $\left\{s_{1}, s_{2}, s_{3}\right\}$. The set of realizable sets $\operatorname{Rel}\left(G^{\prime}\right)$ is exactly the collection $C$. The set of attacks $\left\{s_{1}, s_{2}\right\}$ is critical because every path from $s_{0}^{\prime}$ to the success state $s_{s}^{\prime}$ uses at least one edge with the label in the set $\left\{s_{1}, s_{2}\right\}$. Moreover, $\left\{s_{1}, s_{2}\right\}$ is a hitting set for the collection $C=\left\{C_{1}, C_{2}, C_{3}\right\}$.

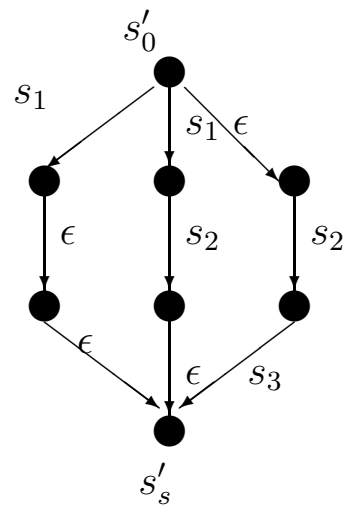

Figure 7. Attack graph corresponding to the collection $C$. 